\title{
The Agreement between the Generalized $p$ Value and Bayesian Evidence in the One-Sided Testing Problem
}

\author{
Yuliang Yin and Bingbing Wang \\ School of Economics, Beijing Technology and Business University, Beijing 100048, China \\ Correspondence should be addressed to Yuliang Yin; imyyl@163.com
}

Received 12 February 2016; Accepted 11 April 2016

Academic Editor: Shey-Huei Sheu

Copyright (C) 2016 Y. Yin and B. Wang. This is an open access article distributed under the Creative Commons Attribution License, which permits unrestricted use, distribution, and reproduction in any medium, provided the original work is properly cited.

In the problem of testing one-sided hypotheses, a frequentist may measure evidence against the null hypothesis by the $p$ value, while a Bayesian may measure it by the posterior probability that the null hypothesis is true. In this paper, we consider the relationship between the generalized $p$ value and the Bayesian evidence in testing one-sided hypotheses in the presence of nuisance parameters. The sufficient conditions for the agreement between these two kinds of evidence are given. Some examples are provided to show the agreement of Bayesian and frequentist evidence in many classical testing problems. This is an illustration of reconcilability of evidence in a general framework where the nuisance parameters are present.

\section{Introduction}

In testing a statistical hypothesis $H_{0}$, Lindley [1] illustrated the possible discrepancy between the Bayesian and frequentist evidence. The relationship between these two kinds of evidence is then extensively studied in the literature. Some important references on this topic include Bartlett [2], Edwards et al. [3], Pratt [4], Dickey [5], Shafer [6], Berger and Delampady [7], Berger and Sellke [8], Meng [9], and Micheas and Dey $[10]$.

For the one-sided testing problem

$$
\begin{aligned}
H_{0}: \theta & \leq \theta_{0} \\
\text { versus } H_{1}: \theta & >\theta_{0},
\end{aligned}
$$

the Bayesian evidence is typically given by the posterior probability of $H_{0}$ and the frequentist evidence is given by the $p$ value. For the situation of testing a location parameter, Casella and Berger [11] considered testing hypotheses (1) based on observing $X=x$, where $X$ has a location density $f(x-\theta)$. Under the assumptions that $f(\cdot)$ is symmetric about zero and that $f(x-\theta)$ has monotone likelihood ratio, it is showed that the lower bound of the posterior probability of $H_{0}$ is equal to the corresponding $p$ value for many classes of prior distributions.
This means that the Bayesian and frequentist evidence are reconcilable in the situation of testing one-sided hypotheses of a location parameter. However, this is not a very general result on the agreement between the Bayesian and frequentist evidence which can cover more testing situations. The relationship of evidence in testing a scale parameter or other parameters is not considered. More generally, it does not consider the situation where nuisance parameters are present. However, the presence of nuisance parameters is very common in practice. For example, we are frequently confronted with the problem of testing a location parameter in the presence of an unknown scale parameter.

In the presence of nuisance parameters, Yin [12] derived the equality of the generalized $p$ value and Bayesian posterior probability of the null hypothesis in the one-sided testing problem under the exponential distribution. However, this is also a result of agreement of evidence in quite specific situation. In this paper, we focus on the one-sided testing problem and study the relationship between the Bayesian and frequentist evidence in a more general setting where the presence of nuisance parameters is allowed. The sufficient conditions for the equivalence between the Bayesian and frequentist evidence are, respectively, given for the one sample and two (or more) samples testing situations. 
This paper is organized as follows. In Section 2, we give the main results on the agreement between the Bayesian and frequentist evidence in the one-sided testing problem. Section 3 illustrates the proposed method by applying it to several classical examples of testing one-sided hypotheses. Conclusions are stated in Section 4.

\section{Agreement of Evidence}

In the presence of nuisance parameters, we consider testing one-sided hypotheses in (1) based on a random sample $X=\left(X_{1}, X_{2}, \ldots, X_{n}\right)$. However, the classical $p$ value is typically not available when nuisance parameters are present. Tsui and Weerahandi [13] introduced the concept of the generalized $p$ value which appears to be useful in situations where conventional frequentist approaches do not provide appropriate measure of evidence.

Let $X=\left(X_{1}, X_{2}, \ldots, X_{n}\right)$ be a random sample distributed with distribution function $F(x ; \zeta)$, where $\zeta=(\theta, \delta)$ is an unknown vector in parameter space $\Omega, \theta \in \Theta$ is a real-valued parameter of interest, and $\delta \in \Delta$ is the nuisance parameter. Assume that $x \in \chi$ is the observed value of $X$.

Definition 1. Let $T=T(X ; x, \zeta)$ be a function of $X, x$, and $\zeta=(\theta, \delta) . T$ is said to be a generalized test variable if it has the following three properties:

(a) $t=T(x ; x, \zeta)$ does not depend on unknown parameters.

(b) When $\theta$ is specified, $T$ has a probability distribution that is free of nuisance parameters.

(c) For fixed $x$ and $\delta, P(T(X ; x, \zeta) \geq T(x ; x, \zeta) \mid \theta)$ is nondecreasing in $\theta$ for any given $t=T(x ; x, \zeta)$.

According to properties (a)-(c) of Definition 1, the larger observed values $T(x ; x, \zeta)$ of $T(X ; x, \zeta)$ can be considered as extreme values of the distribution under the null hypothesis $H_{0}$, so they suggest stronger evidence against $H_{0}$.

Definition 2. Based on a generalized test variable $T=T(X ; x$, $\zeta)$, the generalized $p$ value for testing the one-sided hypotheses (1) is defined as

$$
\begin{aligned}
p(x) & =\sup _{\theta \leq \theta_{0}} P(T(X ; x, \zeta) \geq T(x ; x, \zeta) \mid \theta) \\
& =P\left(T(X ; x, \zeta) \geq T(x ; x, \zeta) \mid \theta=\theta_{0}\right) .
\end{aligned}
$$

Many researches have been carried out to construct the generalized $p$ value for many specific examples including the well-known Behrens-Fisher problem. Hannig et al. [14] provided a general method for constructing the generalized $p$ value under the framework of fiducial inference.

Definition 3. Suppose that there is a random variable $E$ with known distribution on space $\Xi$ and that $h(\zeta, e)$ is a function from $\Omega \times \Xi$ to $\chi$ such that

$$
X=h(\zeta, E)
$$

for every $\zeta \in \Omega$. Furthermore, assume that for any observation $x \in \chi$ of $X$ and $e \in \Xi$ of $E$, the equation $x=h(\zeta, e)$ has a unique solution in $\Omega$ which is denoted by $\zeta_{x}(e)$. Then

(a) the distribution of $\zeta_{x}(E)$ is called the fiducial distribution of $\zeta$ with respect to $x$;

(b) the distribution of $\theta\left(\zeta_{x}(E)\right)$ is called the (marginal) fiducial distribution of $\theta=\theta(\zeta)$ with respect to $x$.

By Definition 3, the fiducial distribution of $\theta=\theta(\zeta)$ is

$$
F_{x}(\theta)=P\left(\theta\left(\zeta_{x}(E)\right) \leq \theta\right) .
$$

Hannig et al. established that if the conditions in Definition 3 hold and if the equation $x=h(\zeta, e)$ has a unique solution in $\Xi$ for any $\zeta$ and $x$, the generalized $p$ value for testing the one-sided hypotheses (1) is just equal to the fiducial $p$ value, $p=F_{x}\left(\theta_{0}\right)$.

For one-sided hypothesis testing problem (1) and in the presence of nuisance parameters, we now give the conditions for the agreement between the frequentist evidence, the generalized $p$ value, and the Bayesian evidence, the posterior probability that $H_{0}$ is true.

Theorem 4. Let $X_{1}, X_{2}, \ldots, X_{n}$ be independently distributed with $F(x ; \xi)$, where $\xi=(\mu, \sigma)$. Suppose that there exit two statistics $T_{1}$ and $T_{2}$ which satisfy

$$
\left(T_{1}, T_{2}\right)=\left(\mu+\sigma E_{1}, \sigma E_{2}\right),
$$

where $E_{1}$ and $E_{2}$ are two independent random variables with a known joint probability density $g\left(e_{1}, e_{2}\right)$, and suppose that the prior distribution of $(\mu, \sigma)$ is $\pi(\mu, \sigma)=1 / \sigma$.

(i) The fiducial distribution of $(\mu, \sigma)$ is equivalent to its posterior distribution.

(ii) For the one-sided testing problem of form (1), where $\theta=$ $\theta(\mu, \sigma)$ is the parameter of interest, the generalized $p$ value is equivalent to the posterior probability of $\mathrm{H}_{0}$.

Proof. (i) On the one hand, since $\left(T_{1}, T_{2}\right)=\left(\mu+\sigma E_{1}, \sigma E_{2}\right)$, we can obtain the functional model as

$$
\begin{aligned}
& T_{1}=\mu+\sigma E_{1}, \\
& T_{2}=\sigma E_{2},
\end{aligned}
$$

based on which we have

$$
\begin{aligned}
& \mu=T_{1}-T_{2} \frac{E_{1}}{E_{2}}, \\
& \sigma=\frac{T_{2}}{E_{2}} .
\end{aligned}
$$

Since $\left(E_{1}, E_{2}\right)$ has a density $g\left(e_{1}, e_{2}\right)$, it can be obtained that the fiducial distribution of $(\mu, \sigma)$ is

$$
f_{t_{1}, t_{2}}(\mu, \sigma)=\frac{t_{2}}{\sigma^{3}} g\left(\frac{t_{1}-\mu}{\sigma}, \frac{t_{2}}{\sigma}\right) .
$$

On the other hand, the density of $\left(T_{1}, T_{2}\right)$ can be obtained as

$$
f\left(t_{1}, t_{2} \mid \mu, \sigma\right)=\frac{1}{\sigma^{2}} g\left(\frac{t_{1}-\mu}{\sigma}, \frac{t_{2}}{\sigma}\right) .
$$


If the prior distribution for $(\mu, \sigma)$ is $\pi(\mu, \sigma)=1 / \sigma$, the posterior distribution of $(\mu, \sigma)$ is

$$
\begin{aligned}
f & \left(\mu, \sigma \mid t_{1}, t_{2}\right) \\
& =\frac{\left(1 / \sigma^{3}\right) g\left(\left(t_{1}-\mu\right) / \sigma, t_{2} / \sigma\right)}{\iint\left(1 / \sigma^{3}\right) g\left(\left(t_{1}-\mu\right) / \sigma, t_{2} / \sigma\right) d \mu d \sigma} \\
& =\frac{t_{2}}{\sigma^{3}} g\left(\frac{t_{1}-\mu}{\sigma}, \frac{t_{2}}{\sigma}\right) .
\end{aligned}
$$

By (8) and (10) we know that the fiducial distribution of $(\mu, \sigma)$ is equivalent to its posterior distribution.

(ii) On the one hand, we know by (i) that the posterior probability of $H_{0}$ is equivalent to the corresponding fiducial $p$ value $F_{x}\left(\theta_{0}\right)$, where $F_{x}(\theta)$ denotes the fiducial distribution of $\theta$. On the other hand, under the assumptions of the theorem it is obvious that the conditions of Definition 3 are satisfied and $\left(t_{1}, t_{2}\right)=\left(\mu+\sigma e_{1}, \sigma e_{2}\right)$ has unique solution in the space of $\left(E_{1}, E_{2}\right)$ for any given $\left(t_{1}, t_{2}\right)$ and $(\mu, \sigma)$, so that the generalized $p$ value is equivalent to the fiducial $p$ value $F_{x}\left(\theta_{0}\right)$. This completes the proof.

For the two-sample testing situation, we also have the result on the agreement between the Bayesian and frequentist evidence which we summarize as Theorem 5.

Theorem 5. Let $X_{1}, X_{2}, \ldots, X_{m}$ and $Y_{1}, Y_{2}, \ldots, Y_{n}$ be independently distributed with $F\left(x ; \xi_{1}\right)$ and $G\left(y ; \xi_{2}\right)$, respectively, where $\xi_{1}=\left(\mu_{1}, \sigma_{1}\right)$ and $\xi_{2}=\left(\mu_{2}, \sigma_{2}\right)$. Suppose that there exit four statistics $T_{11}, T_{12}, T_{21}$, and $T_{22}$ which satisfy

$$
\begin{aligned}
& \left(T_{11}, T_{12}, T_{21}, T_{22}\right) \\
& \quad=\left(\mu_{1}+\sigma_{1} E_{11}, \sigma_{1} E_{12}, \mu_{2}+\sigma_{2} E_{21}, \sigma_{2} E_{22}\right),
\end{aligned}
$$

where $E_{11}, E_{12}, E_{21}$, and $E_{22}$ are four independent random variables with a known joint probability density $g\left(e_{11}, e_{12}, e_{21}, e_{22}\right)$, and suppose that the prior distribution of $\left(\mu_{1}, \sigma_{1}, \mu_{2}, \sigma_{2}\right)$ is $\pi\left(\mu_{1}, \sigma_{1}, \mu_{2}, \sigma_{2}\right)=1 /\left(\sigma_{1} \sigma_{2}\right)$.

(i) The fiducial distribution of $\left(\mu_{1}, \sigma_{1}, \mu_{2}, \sigma_{2}\right)$ is equivalent to its posterior distribution.

(ii) For the one-sided testing problem of form (1), where $\theta=\theta\left(\mu_{1}, \sigma_{1}, \mu_{2}, \sigma_{2}\right)$ is the parameter of interest, the generalized $p$ value is equivalent to the posterior probability of $\mathrm{H}_{0}$.
Proof. (i) By the assumptions that

$$
\begin{aligned}
& \left(T_{11}, T_{12}, T_{21}, T_{22}\right) \\
& \quad=\left(\mu_{1}+\sigma_{1} E_{11}, \sigma_{1} E_{12}, \mu_{2}+\sigma_{2} E_{21}, \sigma_{2} E_{22}\right),
\end{aligned}
$$

the functional model can be obtained as

$$
\begin{aligned}
& T_{11}=\mu_{1}+\sigma_{1} E_{11}, \\
& T_{12}=\sigma_{1} E_{12}, \\
& T_{21}=\mu_{2}+\sigma_{2} E_{21}, \\
& T_{22}=\sigma_{2} E_{22} .
\end{aligned}
$$

Consequently, we have

$$
\begin{aligned}
& \mu_{1}=T_{11}-T_{12} \frac{E_{11}}{E_{12}}, \\
& \sigma_{1}=\frac{T_{12}}{E_{12}}, \\
& \mu_{2}=T_{21}-T_{22} \frac{E_{21}}{E_{22}}, \\
& \sigma_{2}=\frac{T_{22}}{E_{22}} .
\end{aligned}
$$

Since $\left(E_{11}, E_{12}, E_{21}, E_{22}\right)$ has a density $g\left(e_{11}, e_{12}, e_{21}, e_{22}\right)$, we can obtain the fiducial distribution of $\left(\mu_{1}, \sigma_{1}, \mu_{2}, \sigma_{2}\right)$ as

$$
\begin{aligned}
& f_{t_{11}, t_{12}, t_{21}, t_{22}}\left(\mu_{1}, \sigma_{1}, \mu_{2}, \sigma_{2}\right) \\
& =\frac{t_{12} t_{22}}{\sigma_{1}^{3} \sigma_{2}^{3}} g\left(\frac{t_{11}-\mu_{1}}{\sigma_{1}}, \frac{t_{12}}{\sigma_{1}}, \frac{t_{21}-\mu_{2}}{\sigma_{2}}, \frac{t_{22}}{\sigma_{2}}\right) .
\end{aligned}
$$

On the other hand, the density of $\left(T_{11}, T_{12}, T_{21}, T_{22}\right)$ is

$$
\begin{aligned}
f & \left(t_{11}, t_{12}, t_{21}, t_{22} \mid \mu_{1}, \sigma_{1}, \mu_{2}, \sigma_{2}\right) \\
& =\frac{1}{\sigma_{1}^{2} \sigma_{2}^{2}} g\left(\frac{t_{11}-\mu_{1}}{\sigma_{1}}, \frac{t_{12}}{\sigma_{1}}, \frac{t_{21}-\mu_{2}}{\sigma_{2}}, \frac{t_{22}}{\sigma_{2}}\right) .
\end{aligned}
$$

Consequently, the posterior distribution of $\left(\mu_{1}, \sigma_{1}, \mu_{2}, \sigma_{2}\right)$ under the prior $\pi\left(\mu_{1}, \sigma_{1}, \mu_{2}, \sigma_{2}\right)=1 /\left(\sigma_{1} \sigma_{2}\right)$ is

$$
\begin{aligned}
f\left(\mu_{1}, \sigma_{1}, \mu_{2}, \sigma_{2} \mid t_{11}, t_{12}, t_{21}, t_{22}\right) & =\frac{\left(1 / \sigma_{1}^{3} \sigma_{2}^{3}\right) g\left(\left(t_{11}-\mu_{1}\right) / \sigma_{1}, t_{12} / \sigma_{1},\left(t_{21}-\mu_{2}\right) / \sigma_{2}, t_{22} / \sigma_{2}\right)}{\iiint \int\left(1 / \sigma_{1}^{3} \sigma_{2}^{3}\right) g\left(\left(t_{11}-\mu_{1}\right) / \sigma_{1}, t_{12} / \sigma_{1},\left(t_{21}-\mu_{2}\right) / \sigma_{2}, t_{22} / \sigma_{2}\right) d \mu_{1} d \mu_{2} d \sigma_{1} d \sigma_{2}} \\
& =\frac{t_{12} t_{22}}{\sigma_{1}^{3} \sigma_{2}^{3}} g\left(\frac{t_{11}-\mu_{1}}{\sigma_{1}}, \frac{t_{12}}{\sigma_{1}}, \frac{t_{21}-\mu_{2}}{\sigma_{2}}, \frac{t_{22}}{\sigma_{2}}\right) .
\end{aligned}
$$

By (15) and (17), it is obtained that the fiducial distribution of $(\mu, \sigma)$ is equivalent to its posterior distribution. (ii) The proof is by analogy with that of (ii) of Theorem 4. 
Note that the result on the agreement between the Bayesian and frequentist evidence in the two samples situation of Theorem 5 can be easily extended to the general situation of $n(n \geq 2)$ samples.

\section{Examples}

3.1. Normal Distribution. The problem of testing parameters of a normal distribution has its wide applicability. Let $X_{1}, X_{2}, \ldots, X_{n}$ be independently distributed according to the normal distribution $N\left(\mu, \sigma^{2}\right)$, where both $\mu$ and $\sigma^{2}$ are unknown. Let

$$
\begin{aligned}
& T_{1}=\bar{X}=\frac{\sum_{i=1}^{n} X_{i}}{n}, \\
& T_{2}^{2}=S^{2}=\frac{\sum_{i=1}^{n}\left(X_{i}-\bar{X}\right)^{2}}{n-1} .
\end{aligned}
$$

We know that $\sqrt{n}\left(T_{1}-\mu\right) / \sigma$ and $(n-1) T_{2}^{2} / \sigma^{2}$ are independently distributed with $N(0,1)$ and $\chi^{2}(n-1)$. Let

$$
\begin{aligned}
& E_{1} \sim N(0,1), \\
& E_{2}^{2} \sim \chi^{2}(n-1)
\end{aligned}
$$

be independent. We have

$$
\left(T_{1}, T_{2}\right)=\left(\mu+\frac{\sigma E_{1}}{\sqrt{n}}, \frac{\sigma E_{2}}{\sqrt{n-1}}\right) .
$$

By Theorem 4 we know that if the noninformative prior distribution for $\pi(\mu, \sigma)=1 / \sigma$ is used, then for any parameter $\theta=\theta(\mu, \sigma)$ of interest in the problem of testing hypotheses in (1) the generalized $p$ value is equivalent to the posterior probability that the null hypothesis is true.

The problem of comparing the parameters of two normal distributions arises in comparing two treatments, products, and so forth. We now consider the two-sample testing situation. Let $X_{1}, X_{2}, \ldots, X_{m}$ and $Y_{1}, Y_{2}, \ldots, Y_{n}$ be independently distributed with the normal distributions $N\left(\mu_{1}, \sigma_{1}^{2}\right)$ and $N\left(\mu_{2}, \sigma_{2}^{2}\right)$, respectively. Let

$$
\begin{aligned}
& T_{11}=\bar{X}=\frac{\sum_{i=1}^{m} X_{i}}{m}, \\
& T_{12}^{2}=S_{1}^{2}=\frac{\sum_{i=1}^{m}\left(X_{i}-\bar{X}\right)^{2}}{m-1}, \\
& T_{21}=\bar{Y}=\frac{\sum_{i=1}^{n} Y_{i}}{n}, \\
& T_{22}^{2}=S_{2}^{2}=\frac{\sum_{i=1}^{n}\left(Y_{i}-\bar{Y}\right)^{2}}{n-1},
\end{aligned}
$$

and let

$$
\begin{aligned}
& E_{11} \sim N(0,1), \\
& E_{12}^{2} \sim \chi^{2}(m-1),
\end{aligned}
$$

TABLE 1: Fiducial (or posterior) distributions for some important parameters of interest under the normal distribution.

\begin{tabular}{lc}
\hline Parameter & Fiducial (or posterior) distribution \\
\hline$c_{1} \mu+c_{2} \sigma$ & $c_{1}\left(\bar{x}-\frac{\sqrt{n-1} s Z}{\sqrt{n} \sqrt{\chi_{n-1}^{2}}}\right)+c_{2} \frac{\sqrt{n-1} s}{\sqrt{\chi_{n-1}^{2}}}$ \\
$\mu_{1}-\mu_{2}$ & $\bar{x}-\bar{y}-\left(\frac{\sqrt{m-1} s_{1} Z}{\sqrt{m} \sqrt{\chi_{m-1}^{2}}}-\frac{\sqrt{n-1} s_{2} Z}{\sqrt{n} \sqrt{\chi_{n-1}^{2}}}\right)$ \\
$\frac{\sigma_{1}}{\sigma_{2}}$ & $\frac{s_{1}^{2}}{s_{2}^{2}} F_{n-1, m-1}$ \\
\hline
\end{tabular}

$$
\begin{aligned}
& E_{21} \sim N(0,1), \\
& E_{22}^{2} \sim \chi^{2}(n-1),
\end{aligned}
$$

which are independent. We then have that $\left(T_{11}, T_{12}, T_{21}, T_{22}\right)$ are sufficient statistics and

$$
\begin{aligned}
& \left(T_{11}, T_{12}, T_{21}, T_{22}\right) \\
& \quad=\left(\mu_{1}+\frac{\sigma_{1} E_{11}}{\sqrt{m}}, \frac{\sigma_{1} E_{12}}{\sqrt{m-1}}, \mu_{2}+\frac{\sigma_{2} E_{21}}{\sqrt{n}}, \frac{\sigma_{2} E_{22}}{\sqrt{n-1}}\right) .
\end{aligned}
$$

By applying Theorem 5, it is obtained that if we use the noninformative prior $\pi\left(\mu_{1}, \sigma_{1}, \mu_{2}, \sigma_{2}\right)=1 /\left(\sigma_{1} \sigma_{2}\right)$, then for any parameter $\theta=\theta\left(\mu_{1}, \sigma_{1}, \mu_{2}, \sigma_{2}\right)$ in testing hypotheses (1), the generalized $p$ value is equivalent to the posterior probability of the null hypothesis being true.

The fiducial (or posterior) distributions for some important parameters of interest are listed in Table 1, based on which we can give the generalized $p$ value which is also the posterior probability of the null hypothesis for testing hypotheses of form (1). In Table $1, Z$ is a standard normal random variable, $\chi_{m-1}^{2}$ and $\chi_{n-1}^{2}$ are chi-squared random variables with the indicated degrees of freedom, $F_{n-1, m-1}$ is $F$-variable with the indicated degrees of freedom, and $c_{1}$ and $c_{2}$ are two constants.

From Table 1 we observe that if $c_{1}=1$ and $c_{2}=0$, the distribution reduces to the fiducial (or posterior) distribution of a normal mean. If $c_{1}=0$ and $c_{2}=1$, the distribution reduces to the fiducial (or posterior) distribution of a normal standard variance. If $c_{1}=1$ and $c_{2}=z_{p}$, the distribution then reduces to the fiducial (or posterior) distribution of the $p$ quantile, where $z_{p}$ is the $p$-quantile of the standard normal distribution.

In addition, by Theorem 4 , an immediate agreement of evidence can be obtained in testing $H_{0}: \theta=\mu / \sigma \leq \theta_{0}$, which is the hypothesis about the mean expressed in $\sigma$-unit, and in testing $H_{0}: \theta=P\left(X \leq x_{0}\right) \leq \theta_{0}$, which is the hypothesis about the distribution function of the normal variable with mean $\mu$ and variance $\sigma^{2}$ at a fixed value $x_{0}$. The generalized $p$ value which is equivalent to the posterior probability of $H_{0}$ can be easily obtained according to Table 1 since these two null hypotheses can be equivalently expressed as $H_{0}^{\prime}$ : $\mu-$ $\theta_{0} \sigma \leq 0$ and $H_{0}^{\prime}:-\mu-\Phi^{-1}\left(\theta_{0}\right) \sigma \leq-x_{0}$, respectively. 
3.2. Two-Parameter Exponential Distribution. We first consider the relationship between the Bayesian and frequentist evidence for testing one-sided hypotheses in the one-sample situation. Let $X_{1}, X_{2}, \ldots, X_{n}$ be independently distributed according to the two-parameter exponential population $E(\mu, \sigma)$, where both $\mu$ and $\sigma$ are unknown. Suppose that the observations are type II censored data $X_{(1)}<X_{(2)}<\cdots<$ $X_{(k)}, k<n$.

We know that

$$
\begin{aligned}
& T_{1}=X_{(1)}, \\
& T_{2}=\sum_{i=1}^{k} X_{(i)}+(n-k) X_{(k)}-n X_{(1)}
\end{aligned}
$$

are jointly sufficient statistics for $(\mu, \sigma)$ and

$$
\begin{gathered}
\frac{2 n\left(X_{(1)}-\mu\right)}{\sigma} \\
\frac{2\left(\sum_{i=1}^{k} X_{(i)}+(n-k) X_{(k)}-n X_{(1)}\right)}{\sigma}
\end{gathered}
$$

are independently distributed with $\chi^{2}(2)$ and $\chi^{2}(2 k-2)$. Let

$$
\begin{aligned}
& E_{1} \sim \chi^{2}(2), \\
& E_{2} \sim \chi^{2}(2 k-2)
\end{aligned}
$$

be independent. We have

$$
\left(T_{1}, T_{2}\right)=\left(\mu+\frac{\sigma E_{1}}{2 n}, \frac{\sigma E_{2}}{2}\right) .
$$

If the prior distribution of $(\mu, \sigma)$ is $\pi(\mu, \sigma)=1 / \sigma$, then according to Theorem 4, for each parameter $\theta=\theta(\mu, \sigma)$ of interest in testing hypotheses (1) under a two-parameter exponential distribution, the generalized $p$ value is equivalent to the corresponding posterior probability of the null hypothesis.

The problem of comparing parameters of two exponential distributions arises in many theoretical and applied contexts. A case in point is the problem of lifetime testing in the theory of reliability. We then begin to consider the relationship between the Bayesian and frequentist evidence in a twopopulation context.

Let $X_{1}, X_{2}, \ldots, X_{m}$ and $Y_{1}, Y_{2}, \ldots, Y_{n}$ be independently distributed with the two-parameter exponential distributions $E\left(\mu_{1}, \sigma_{1}\right)$ and $E\left(\mu_{2}, \sigma_{2}\right)$, respectively, where all the parameters are unknown. The observations are type II censored data $X_{(1)}<X_{(2)}<\cdots<X_{(k)}, k<m$, and $Y_{(1)}<Y_{(2)}<$ $\cdots<Y_{(l)}, l<n$. The prior distribution in the situation of two populations is $\pi\left(\mu_{1}, \sigma_{1}, \mu_{2}, \sigma_{2}\right)=1 /\left(\sigma_{1} \sigma_{2}\right)$.

If we denote

$$
\begin{aligned}
& T_{11}=X_{(1)}, \\
& T_{12}=\sum_{i=1}^{k} X_{(i)}+(m-k) X_{(k)}-m X_{(1)},
\end{aligned}
$$

$$
\begin{aligned}
& T_{21}=Y_{(1)}, \\
& T_{22}=\sum_{i=1}^{l} Y_{(i)}+(n-l) Y_{(l)}-n Y_{(1)}
\end{aligned}
$$

and if

$$
\begin{aligned}
& E_{11} \sim \chi^{2}(2), \\
& E_{12} \sim \chi^{2}(2 k-2), \\
& E_{21} \sim \chi^{2}(2), \\
& E_{22} \sim \chi^{2}(2 l-2),
\end{aligned}
$$

which are independent, then we have that $\left(T_{11}, T_{12}, T_{21}, T_{22}\right)$ are sufficient statistics and

$$
\begin{aligned}
& \left(T_{11}, T_{12}, T_{21}, T_{22}\right) \\
& \quad=\left(\mu_{1}+\frac{\sigma_{1} E_{11}}{2 m}, \frac{\sigma_{1} E_{12}}{2}, \mu_{2}+\frac{\sigma_{2} E_{21}}{2 n}, \frac{\sigma_{2} E_{22}}{2}\right) .
\end{aligned}
$$

By Theorem 5, for any parameter $\theta=\theta\left(\mu_{1}, \sigma_{1}, \mu_{2}, \sigma_{2}\right)$ of interest in one-sided testing problem (1), the equivalence between the generalized $p$ value and the posterior probability of the null hypothesis being true can be obtained.

Table 2 lists the fiducial (or posterior) distributions for some important parameters of interest under the twoparameter exponential distribution. The generalized $p$ value which is also the posterior probability of the null hypothesis for testing one-sided hypotheses (1) can be easily obtained according to the corresponding distribution in Table 2, where $\chi_{2}^{2}, \chi_{2 k-2}^{2}$, and $\chi_{2 l-2}^{2}$ are chi-squared random variables with the indicated degrees of freedom, $F_{2 l-2,2 k-2}$ is a $F$-variable with the indicated degrees of freedom, and $c_{1}$ and $c_{2}$ are two constants.

Note from Table 2 that if $c_{1}=1$ and $c_{2}=0$, the distribution reduces to the fiducial (or posterior) distribution for testing the location parameter $\mu$. If $c_{1}=0$ and $c_{2}=1$, the distribution reduces to the fiducial (or posterior) distribution for testing the scale parameter $\sigma$.

In the theory of reliability, a parameter of particular interest under a two-parameter exponential distribution is often a quantile of the form $\mu+b \sigma$, where $b$ is a known and fixed constant. The agreement between the generalized $p$ value and the Bayesian evidence for testing this quantile can be obtained by Theorem 5 and the evidence can be given easily according to Table 2.

Moreover, the agreement between the Bayesian and frequentist evidence for testing the reliability function $\theta=$ $P\left(X \geq x_{0}\right)=\exp \left\{-\left(x_{0}-\mu\right) / \sigma\right\}$, where $x_{0}$ is a fixed value, can be obtained by Theorem 5 . Since the hypothesis $H_{0}: \exp \left\{-\left(x_{0}-\mu\right) / \sigma\right\} \leq \theta_{0}$ can be equivalently expressed as $H_{0}^{\prime}: \mu-\ln ^{\theta_{0}} \sigma \leq x_{0}$, the generalized $p$ value which is equivalent to the posterior probability of the null hypothesis can be obtained easily according to Table 2 . 
TABLE 2: Fiducial (or posterior) distribution for some important parameters of interest under the two-parameter exponential distribution.

\begin{tabular}{lc}
\hline Parameter & Fiducial (or posterior) distribution \\
$c_{1} \mu+c_{2} \sigma$ & $c_{1} x_{(1)}-\frac{c_{1}\left[\sum_{i=1}^{k} x_{(i)}+(n-k) x_{(k)}-n x_{(1)}\right] \chi_{2}^{2}}{n \chi_{2 k-2}^{2}}+\frac{2 c_{2}\left[\sum_{i=1}^{k} x_{(i)}+(n-k) x_{(k)}-n x_{(1)}\right]}{\chi_{2 k-2}^{2}}$ \\
$\mu_{1}-\mu_{2}$ & $x_{(1)}-y_{(1)}+\frac{\sum_{i=1}^{l} y_{(i)}+(n-l) y_{(l)}-n y_{(1)}}{n} \frac{\chi_{2}^{2}}{\chi_{2 l-2}^{2}}-\frac{\sum_{i=1}^{k} x_{(i)}+(m-k) x_{(k)}-m x_{(1)}}{m} \frac{\chi_{2}^{2}}{\chi_{2 k-2}^{2}}$ \\
$\frac{\sigma_{1}}{\sigma_{2}}$ & $\frac{(l-1)\left[\sum_{i=1}^{k} x_{(i)}+(m-k) x_{(k)}-m x_{(1)}\right]}{(k-1)\left[\sum_{i=1}^{l} y_{(i)}+(n-l) y_{(l)}-n y_{(1)}\right]} F_{2 l-2,2 k-2}$ \\
\hline
\end{tabular}

3.3. Weibull Distribution. In the theory of survival analysis and the theory of reliability, one of the most important distributions is the Weibull distribution $W(\tau, \eta)$ whose density is

$$
f(x)=\frac{\tau x^{\tau-1}}{\eta^{\tau}} e^{-x^{\tau} / \eta^{\tau}}, \quad x>0,
$$

where $\tau>0$ and $\eta>0$.

Suppose that $X \sim W(\tau, \eta)$ and let $Y=\ln X$, then the density of $Y$ is

$$
f(y)=\frac{1}{\sigma} e^{(y-\mu) / \sigma} e^{-e^{(y-\mu) / \sigma}}
$$

where $\mu=\ln \eta$ and $\sigma=1 / \tau$. We know that (32) is the density of the extreme value distribution which is denoted by $\operatorname{EV}(\mu, \sigma)$. It can be verified that $E(Y)=\mu-r \sigma$ and $\operatorname{Var}(Y)=$ $\pi^{2} \sigma^{2} / 6$, where $r$ is the Euler constant.

Note that it is more convenient to make inferences on the parameters of a Weibull distribution under (32) since it is a location-scale family. Let $X_{1}, X_{2}, \ldots, X_{n}$ be independently distributed according to the Weibull distribution $W(\tau, \eta)$ and let $Y_{i}=\ln X_{i}, i=1,2, \ldots, n$. Then $Y_{1}, Y_{2}, \ldots, Y_{n}$ are independently distributed according to the density (32). Let

$$
\begin{aligned}
& T_{1}=\bar{Y}=\frac{\sum_{i=1}^{n} Y_{i}}{n}=\frac{\ln \prod_{i=1}^{n} X_{i}}{n}, \\
& T_{2}^{2}=S^{2}=\frac{\sum_{i=1}^{n}\left(Y_{i}-\bar{Y}\right)^{2}}{n-1},
\end{aligned}
$$

and let

$$
\begin{aligned}
& E_{1}=\frac{\sqrt{6}\left[T_{1}-(\mu-r \sigma)\right]}{\pi \sigma}, \\
& E_{2}=\frac{\sqrt{6} T_{2}}{\pi \sigma},
\end{aligned}
$$

and then we have

$$
\left(T_{1}, T_{2}\right)=\left(\mu+\sigma\left(\frac{\pi E_{1}}{\sqrt{6}}+r\right), \sigma \frac{\pi E_{2}}{\sqrt{6}}\right) .
$$

If the prior distribution for $(\mu, \sigma)$ is $\pi(\mu, \sigma)=1 / \sigma$, then according to Theorem 4 , we can obtain the equivalence between the generalized $p$ value and the posterior probability of the null hypothesis for the problem of testing hypotheses about $\theta=\theta(\mu, \sigma)=\theta^{*}(\tau, \eta)$. But we have to note that $\left(T_{1}, T_{2}\right)$ is not sufficient for $(\mu, \sigma)$ in this situation and therefore may lead to some loss of information about the parameters which is contained in the sample.

Now we consider the two-sample situation. Let $X_{11}, X_{12}, \ldots, X_{1 m}$ and $X_{21}, X_{22}, \ldots, X_{2 n}$ be independently distributed with the Weibull distributions $W\left(\tau_{1}, \eta_{1}\right)$ and $W\left(\tau_{2}, \eta_{2}\right)$, respectively. Let $Y_{1 i}=\ln X_{1 i}, i=1,2, \ldots, m$, and $Y_{2 j}=\ln X_{2 j}, j=1,2, \ldots, n$. Then $Y_{11}, Y_{12}, \ldots, Y_{1 m}$ and $Y_{21}, Y_{22}, \ldots, Y_{2 n}$ are independently distributed with the extreme value distributions $\operatorname{EV}\left(\mu_{1}, \sigma_{1}\right)$ and $\operatorname{EV}\left(\mu_{2}, \sigma_{2}\right)$, respectively, where $\mu_{i}=\ln \eta_{i}, i=1,2$, and $\sigma_{j}=1 / \tau_{j}, j=1,2$. Now let

$$
\begin{aligned}
& T_{11}=\overline{Y_{1}}=\frac{\sum_{i=1}^{m} Y_{1 i}}{m}=\frac{\ln \prod_{i=1}^{m} X_{1 i}}{m}, \\
& T_{12}^{2}=S_{1}^{2}=\frac{\sum_{i=1}^{m}\left(Y_{1 i}-\overline{Y_{1}}\right)^{2}}{m-1}, \\
& T_{21}=\overline{Y_{2}}=\frac{\sum_{i=1}^{n} Y_{2 i}}{n}=\frac{\ln \prod_{i=1}^{n} X_{2 i}}{n}, \\
& T_{22}^{2}=S_{2}^{2}=\frac{\sum_{i=1}^{n}\left(Y_{2 i}-\overline{Y_{2}}\right)^{2}}{n-1},
\end{aligned}
$$

and let

$$
\begin{aligned}
& E_{11}=\frac{\sqrt{6}\left[T_{11}-\left(\mu_{1}-r \sigma_{1}\right)\right]}{\pi \sigma_{1}}, \\
& E_{12}=\frac{\sqrt{6} T_{12}}{\pi \sigma_{1}}, \\
& E_{21}=\frac{\sqrt{6}\left[T_{21}-\left(\mu_{2}-r \sigma_{2}\right)\right]}{\pi \sigma_{2}}, \\
& E_{22}=\frac{\sqrt{6} T_{22}}{\pi \sigma_{2}},
\end{aligned}
$$


and then we have

$$
\begin{aligned}
& \left(T_{11}, T_{12}, T_{21}, T_{22}\right)=\left(\mu_{1}\right. \\
& \quad+\sigma_{1}\left(\frac{\pi E_{11}}{\sqrt{6}}+r\right), \sigma_{1} \frac{\pi E_{12}}{\sqrt{6}}, \mu_{2} \\
& \left.\quad+\sigma_{2}\left(\frac{\pi E_{21}}{\sqrt{6}}+r\right), \sigma_{2} \frac{\pi E_{22}}{\sqrt{6}}\right) .
\end{aligned}
$$

Consequently, we can obtain the equivalence between the Bayesian and frequentist evidence in testing one-sided hypotheses about $\theta=\theta\left(\mu_{1}, \sigma_{1}, \mu_{2}, \sigma_{2}\right)=\theta^{*}\left(\tau_{1}, \eta_{1}, \tau_{2}, \eta_{2}\right)$ of interest by Theorem 5 given that the prior distribution for $\left(\mu_{1}, \sigma_{1}, \mu_{2}, \sigma_{2}\right)$ is $\pi\left(\mu_{1}, \sigma_{1}, \mu_{2}, \sigma_{2}\right)=1 /\left(\sigma_{1} \sigma_{2}\right)$.

\section{Conclusions}

For the one-sided testing problem, we give the sufficient conditions for the equivalence between the generalized $p$ value and the posterior probability that the null hypothesis is true. By applying the proposed method to some specific examples we show the agreement between the Bayesian and frequentist evidence in many classical testing situations. This is an illustration of reconcilability of the Bayesian and frequentist evidence in the one-sided testing problems under a quite general framework where the presence of nuisance parameters is allowed.

For the testing problems we have considered in this paper, the posterior distribution of the parameter of interest is equivalent to the corresponding fiducial distribution, which is basically the main reason for the equivalence between the generalized $p$ value and the posterior probability of the null hypothesis. In the problem of testing a normal standard variance, we have that

$$
\sigma \mid x \sim \sqrt{n-1} s\left(\chi_{n-1}^{2}\right)^{-1 / 2},
$$

where $\chi_{n-1}^{2}$ is a chi-squared random variable with $n$ - 1 degrees of freedom. This means that we can give a constructive form of the posterior distribution for a parameter of interest since we have a general method of formulating a fiducial distribution. This kind of constructive form of distributions may bring us significant convenience in computation and simulation. If the conditions for the agreement of evidence hold for a certain distribution or a family of distributions, the corresponding fiducial (or posterior) distribution table like Table 1 or Table 2 can be given for the convenience of use in theory and practice.

\section{Competing Interests}

The authors declare that they have no competing interests.

\section{References}

[1] D. V. Lindley, "A statistical paradox," Biometrika, vol. 44, pp. 187-192, 1957.
[2] M. S. Bartlett, "A comment on D. V. Lindley's statistical paradox," Biometrika, vol. 44, no. 3-4, pp. 533-534, 1957.

[3] W. Edwards, H. Lindman, and L. J. Savage, "Bayesian statistical inference for psychological research," Psychological Review, vol. 70, pp. 193-242, 1963

[4] J. W. Pratt, "Bayesian interpretation of standard inference statements (with discussion)," Journal of the Royal Statistical Society, Series B, vol. 27, pp. 169-203, 1965.

[5] J. M. Dickey, "Is the tail area useful as an approximate Bayes factor?" Journal of the American Statistical Association, vol. 72, no. 357, pp. 138-142, 1977.

[6] G. Shafer, "Lindley's paradox," Journal of the American Statistical Association, vol. 77, no. 378, pp. 325-351, 1982.

[7] J. O. Berger and M. Delampady, "Testing precise hypotheses (with discussion)," Statistical Science, vol. 2, pp. 317-352, 1987.

[8] J. O. Berger and T. Sellke, "Testing a point null hypothesis: the irreconcilability of $p$-values and evidence," Journal of the American Statistical Association, vol. 82, no. 397, pp. 112-122, 1987.

[9] X.-L. Meng, "Posterior predictive p-values," The Annals of Statistics, vol. 22, no. 3, pp. 1142-1160, 1994.

[10] A. C. Micheas and D. K. Dey, "Reconciling Bayesian and frequentist evidence in the one-sided scale parameter testing problem," Communications in Statistics-Theory and Methods, vol. 36, no. 6, pp. 1123-1138, 2007.

[11] G. Casella and R. L. Berger, "Reconciling Bayesian and frequentist evidence in the one-sided testing problem," The Journal of the American Statistical Association, vol. 82, no. 397, pp. 106-111, 1987.

[12] Y. Yin, "Generalized p-values and Bayesian evidence in the one-sided testing problems under exponential distributions," Statistica Neerlandica, vol. 65, no. 3, pp. 319-336, 2011.

[13] K.-W. Tsui and S. Weerahandi, "Generalized $p$-values in significance testing of hypotheses in the presence of nuisance parameters," Journal of the American Statistical Association, vol. 84, no. 406, pp. 602-607, 1989.

[14] J. Hannig, H. Iyer, and P. Patterson, "Fiducial generalized confidence intervals," Journal of the American Statistical Association, vol. 101, no. 473, pp. 254-269, 2006. 


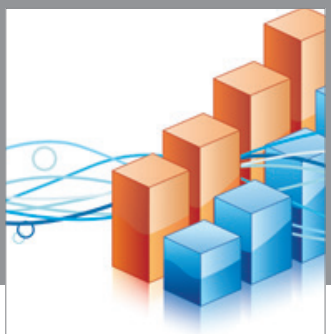

Advances in

Operations Research

vatem alat4

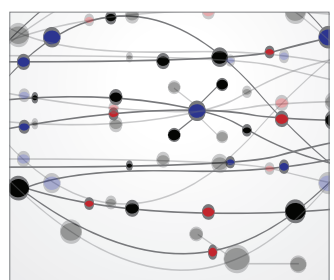

\section{The Scientific} World Journal
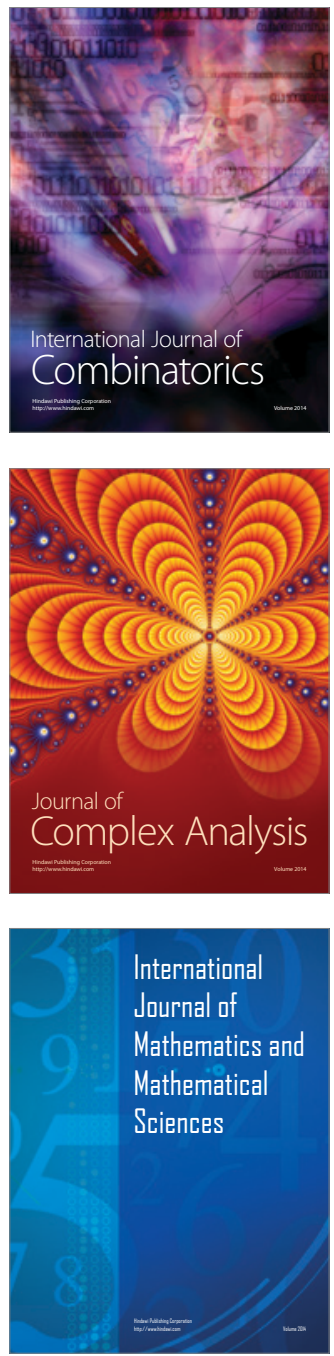
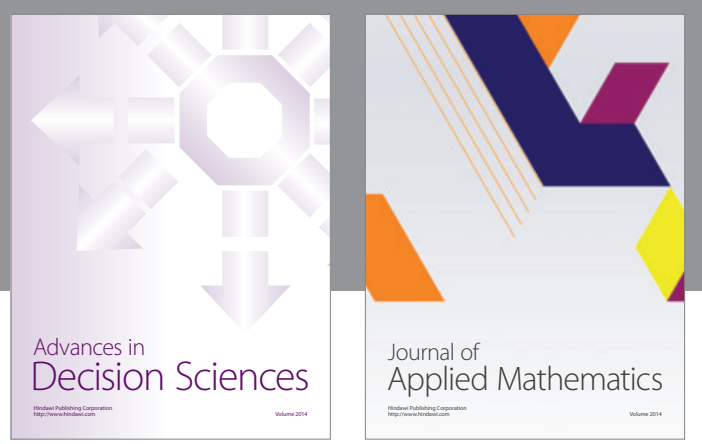

Algebra

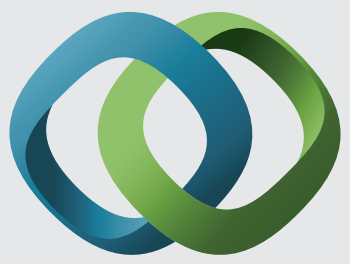

\section{Hindawi}

Submit your manuscripts at

http://www.hindawi.com
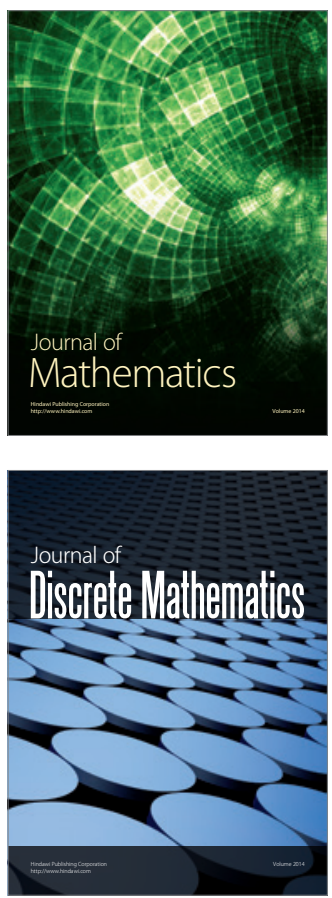

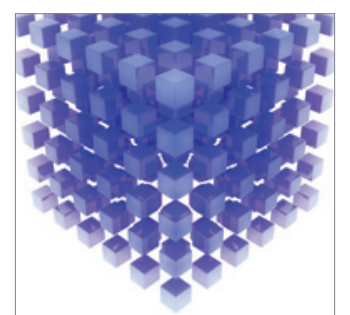

Mathematical Problems in Engineering
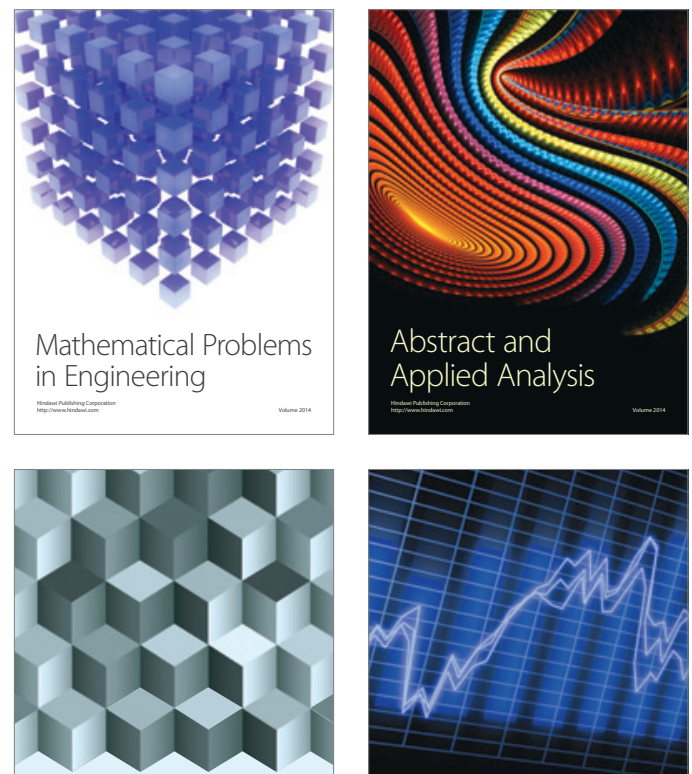

Journal of

Function Spaces

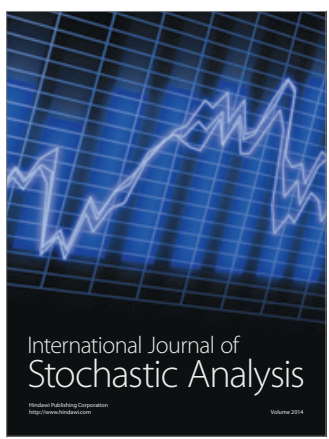

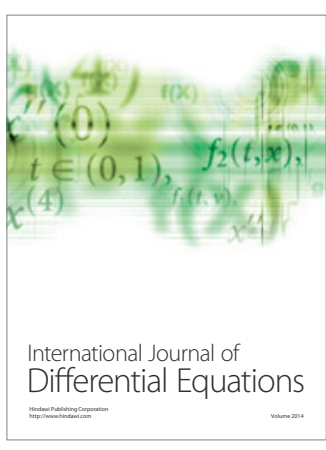
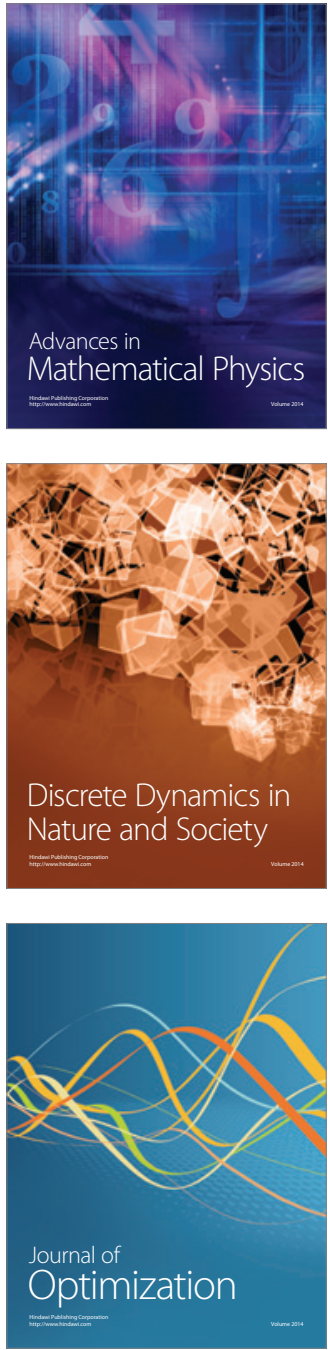